\title{
Sobre la penetración de José 1 en Hispanoamérica
}

\author{
Pot Daniel VALCÁRCel
}

Se ha puesto en tela de juicio la concreta penetración de Agentes del rey José I, instrumento de Napoleón para el gobierno de España y las Indias. Los documentos inéditos : 1) Instrucciones y II) Rol de Comisionados - más abajo insertos-., permitirán formar opinión adecuada y ser punto de partida para una investigación sistemática y rectificatoriá de calgunas bpintones tradicionales.

Desde la renoyadora época de carlos III se formó un pequeño pero selecto "grupo progresista, puesto de lado durante el gobierno de Carlos IV. Cuando se produjo la invasión napoleónica, algunos de sus componentes pensaron que José Bonaparte podría ser el instrumento para alcanzar las mejoras sociales, económicas y políticas a que aspiraban. En consecuencia, tomaron el partido de José I desde 1808, recibiendo de inmediato el mote de afrancesados. Pero los ultramontanos, con buena parte de los liberales y el pueblo adoptó el partido contrario, por no ver otra cosa que la momentánea política. Los intentos de José Napoleón por conquistar el favor de los españoles, tienen una muestra en la Nueva Constitucion q. ha de regir en España, e Yndias aprobada por la Junta española en Bayona, el seis de julio de 1808. Una minuciosa confrontación crítica entre el texto de la Constitución de Bayona (1808) y la Constitución de Cádiz (1812), permitiría arribar a ciertas conclusiones de importancia para una interpretación renovada. 
Aunque se ha dicho que la penetración francesa fue un simple conato, su concreta presencia histórica podría divisarse:

I) Con el texto de las Instrucciones dadas por José I a su

Comisionado o Agente principal Mr. Desmolard, residente en Baltimore, y "á los demas q. baxo sus ordenes han pasado á las Americas Españolas con el fin de sublevarlas", seguidas de una Nota de distinto origen, añadida en Caracas por Juan Germán Roscio. La gopia se halla'en el Public Record Office de Londres, F.O. 72/104, ff. 178-179 (trad. inglesa: ibid ff. 180-187), a base del texto que existió en la Secretaría de la Suprema Junta Conservadora de los Derechos de Fernando VII de Caracas, firmada por el Vocal de la Junta y Secretario de Relaciones Exteriores J. G. Roscio en 31-V-1810.

Según las citadas Instrucciones, Baltimore era el centro de la actividad francesa dirigida por Mr. Desmolard. Se debía manifestar a los Criollos que el principal objetivo de José I era la independencia hispanoamericana, con el auxilio bélico necesario que Estados Unidos Te habría ofrecido. Cada Comisario o Agente Jefe, con conocimiento del terreno y medio social, escogería los colaboradores apropiadós para propagar las ventajas de la independencia, señalar las desventajas del enorme caudal periódica-

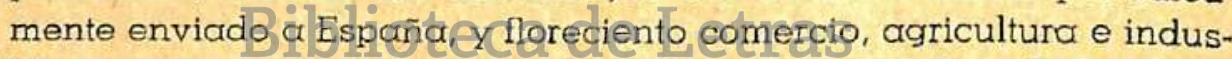
trias que se adquirirían con la independencia. Los Agentes tenían asimismo consigna de vincularse con toda clase de autoridades y gente principal -especialmente eclesiástica-., y difundir la misión divina de Napoleón. Entre los Criollos había que recalcar su injusta situación frente a la de los europeos, cuyo lógico fruto era notorio al comparar la situación de Hispanoamérica con la de los Estados Unidos. No se atacaría sino defendería a la Inquisición y al Clero. El lema de los futuros estandartes de la revolución sería: viva la Religión Católico Apostólica Romana, y muera el mal Gobierno. Manifestarían a los Indios las ventajas de ser nuevamente dueños de sus países, gracias al auxilio de Napoleón. El estallido simultáneo de la revolución, debía prepararse con sumo cuidado y coordinarse en las diversas regiones. Por las vías que se indicasen, serían remitidos partes periódicos a los Agentes y éstos al Comisionado principal en Baltimore, confeccionándose y completándose el rol de los partidarios de la independencia hispanoamericana. Como medida inmediata, se 
aconseja impedir el envío de caudales mediante los Comisionados on diversos puertos hispanoamericanos.

La Nota complementaria, añadida en Caracas por J. G. Roscio, proporciona datos de gran interés: alistamiento de tres goletas en Baltimore, viaje de cuatro buques a puertos diversos del continente (México, Nueva Granada, Buenos Aires y Perú), noticia del gran número de partidarios criollos principalmente en México y de algunos poderosos Gobernadores indígenas, y asimismo en Lima.

II) Con el Rol de Comisionados de José I, copia existente en el Public Record Office, F. $0.72 / 104$, ff. 188-189v. (trad. inglesa: ibid ff. 190-195). Es una lista de los Agentes en los diversos territorios hispánicos de América, con especificación de origen y radio geográfico de acción, lista tomada también de la Secretaría de la Suprema Junta Conservadora de los Derechos de Fernando VII en Caracas y firmada asimismo por J. G. Roscio en 1-VI-1810.

Los documentos insertos han sido estudiados y trascritos con ayuda del Instituto Panamericano de Geografía e Historia de México y colaboración de Unesco.

Copia de las instrucciones dadas por el Usurpador Jph. Napoleon al Comisionado, ó Agente princfpal q. tiene en Baftimore Mr. Desmolard, y á los demas q. baxo sus ordenes han pasado á las Americas Españolas con el fin de sublevarlas

El objeto que deberan pr ahora proponerse estos Comisionados no es otro q. el de manifestar á los Criollos de America, y persuadirlos que S.M.Y. Y R. no desea otra cosa, sino dar la libertad á un Pueblo esclavo de tantos años sin mas recompensa pr tan alto beneficio, q. la amistad de los naturales y el comercio de los Puertos en ambas Americas = Que pa q. sea libre, é independiente de Europa ofrece su dicha Magestad todos los auxilios q. sean necesarios, quiere decir tropa, y pertrechos de guerra, sobre cuyos puntos está yá de acuerdo con los Estados Unidos de America q. están prontos á facilitarselos = Cada Comisario, ó Agente en Xefe como instruido del terreno en q. se halla colocado, y del caracter de sus habitantes sabrá escoger sugetos capaces pa en- 
cargarles de los asuntos q. convengan, pa persuadir al Pueblo, y hacerle ver las ventajas q. le resultan de sacudir el yugo Europeo $=$ Se hará ver los caudales q. permaneceran, y giraran en las Americas, suspendiendo las crecidas remesas que continuamente se remiten á España, el aumento q. tendrá su comercio con la libertad de sus Puertos pa todas las Naciones estrangeras, las ventajas q. resultarán á la Nacion de la libre agricultura, sembrancomo es la cultura del azafrán, lino, cañamo, el hacer aceyte, las viñas $\&=E l$ beneficio que sacarán del establecimto de fabricas de toda especie = La gran satisfacción, y ventajas q. sacaran dhos. Pueblos de la abolicion del estanco del tavaco, polbora \&. y del papel sellado $=$ Para conseguir todo esto con facilidad, como el pueblo es por la mayor parte barbaro deberan antes todas cosas los Comisionados hacerse estimar de los Gobernadores, Yntendtes Subdelgdos de los Curas Parrocos, y Prelados Religiosos, no causaran gastos ni medio alguno para lograr sus amistades, en particular con los Eclesiasticos, procurando q. estos en las Confesiones persuadan, y aconsejen á los Penitentes q. les conviene un Gobierno independiente, y q. no deben perder una ocasion tan oportuna como la q. se les presenta, y facilita al Emperador Napoleon, haciendoles creer q. es enviado pr la mano de Dios (ff. 178v.) para castigar el orgullo, y tirania de los Monarcas, que es un pecado mortálo y 19 . he admite perdort-el resistirse á la voluntad Divina $=$ Recordarán á estos en todos los momentos la oposicion q. les tienen "los Europeos, el vil trato q. les"dán, y el desprecio con q. los tratan = Recordaran puntualmte á los Yndios las crueldades q. los Españoles usaron en sus conquistas, y las injusticias, é infamias q. cometieron con sus legitimos Soberanos, destronandolos, quitandoles la vida, y esclavizandolos = Les pondrán á la vista las injusticias $q$. experimentan diariamente en sus solicitudes á empleos, los quales se proveen pr sus Virreyes, y Gobernadores en aquellos q. presentan mas empeños, ó dinero dexando al meritorio abandonado = Les harán patente el crecido numero de habitantes de las Provincias en donde se hallen, el de los hombres de merito, y de los empleados tanto en la carrera eclesiastica, como en la secular, donde hallaran bien clara la injusticia, cotejanla diferencia de los talentos, y meritos de los criollos con los de los empleados Europeos = Les manifestarán la diferencia q. hay entre los Estados Unidos, y las Americas Españolas; la satisfaccion de q. gozan estos Americanos, sus progresos en el comercio, 
y navegacion, y el gusto con q. viven libres del yugo Europeo; solamte con su gobierno patriotico, y electivo, y les aseguran $q_{4}$ siendo libres las Americas de España serán las Legisladoras del Universo = Cada Comisionado, tanto los en Xefe como los demas deberán apuntar los nombres de los que se declaren Amigos, y miembros de la libertad, los subalternos remitiran sus listas á los en Xefe, y estos al Enviado mio á los Estados Unidos po mi gobierno y recompensa debida á cada individuo $=$ Se abstendran mis Comisionados de hablar contra la Ynquisicion, ni estado Eclesiastico, antes bien deberan en sus conversaciones apoyar la necesidad de aquel Santo Tribunal, y el provecho del segundo = En los Estandartes, ó banderas de la sublevación irá escrito el mote de viva la Religion Catolica Apostolica Romana, y muera el mal gobierno = Manifestarán á los Yndios quanta satisfaccion sera pa ellos ser dueños otra vez de su-Pais, y libres del tirano tributo q. dan á un estrangero Monarca $=\mathrm{Y}$ ultimamte les advertiran $\mathrm{q}$. este no existe (ff.179) en su Gobierno sino en el poder del restaurador de la libertad y Legislador Universal Napoleon = En fin dhos. Comisionados deberan procurar pr todos los medios posibles hacer patente al Pueblo la utilidad a. se le origina con dho. Gobierno = Despues de tener yá en punto la revolucion, alistados todos los miembros principales que deben entrar en ella de cada Ciudad, y Prova será quando los primeros Xefes, y Comisionados deberán saver convinar, y ver la mayor ocasion pd la sublevacion, pasando avisos en tiempo ar bos deinasinsubaltemos, preque se verifique esta en un mismo dia, y hora en los diferentes puntos $q$. convengue, pues este es el principal punto, y el q. debe facilitar la empre$\mathrm{s} a=$ Los Comisionados en Xefe en cada una de las Provincias de su departamto y los Subalternos q. les estan señalados, tendrán de su parte á los familiares de los Gobernadores, Yntendentes, y demas Xejes, y pr medio de estos envenenarán á los de estas clases q. consideren rebeldes á la empresa, lo qual se deberá executar antes de la revolución pa quitar de en medio estos estorbos $=$ El primer punto que se tratará será el de impedir las remesas de caudales á la Peninsula, lo q. se verificará facilmte teniendo buenos Comisionados en Vera Cruz, y demas Puertos del Continente, siendo el primero Vera Cruz, en donde todos los buques q. llegaren de Europa serán recibidos, y al instante su tripulacion, y oficialidad puestas en las fortalezas, impidiendo la salida de todo buque en general hasta el buen exito, y adelantamto de la revolucion = 
Se encarga á los Comisionados el q. los Subalternos den repetidos partes de los progresos de esta sublevacion de su cargo á los Xefes, y estos al Enviado en los Estados Unidos pr las vias que se les señalen = Para este efecto se procurará tener lista la correspondencia pr tierra hasta los puntos que se halle pr conveniente de la Costa donde siempre habrá buques prontos po qualquier evento $=$

(fdo.) Jph. Napoleon $=$ A mi Enviado Desmolard.

Nota $=A$ el consabido objeto se están alistando otras tres Goletas mas en Baltimore = Actualmente hay quatro buques q. viajan á diferentes puntos del Continente como saben los Comisionados, pr donde continuarán dando parte de las ocurrencias. Los puntos que (ff.179v.) estos freqüenten mas particularmte son Nuevo Santander, Tampico, en el Reyno de Mexico, la Costa de Comayagua por Trugillo, en Guatemala, y Puertos del Peru, Cumaná, Rio de la Hacha \&. po Cartagena, Santa Fe, Caracas, y demas, Costa Firme donde andan froquentemte dos buques como contrabandistas de Jamayca = Asegura el Enviado Desmolard q. segun avisos de Mexico, llegados reciemte es infinito el numero de partidarios q. se hallan ya alistados, todos del primer rango, y está muy confiado de a. lacerreresa secverificarázen aquel Reyno, y q. en el punto de Vera Cruz tiene por segura la execucion de su pro. yecto, y q. este sera el punto de vista de toda la expedicion = Que tiene pronto para el caso, un conducto seguro po avisar á Nueva Orleans, en donde están prontos todos los socorros necesarios pero q. aun estos le parece q. seran inutiles segun las apariencias de felicidad q. prometen tanto el partido q. tiene adquirido, quanto la ineptitud de aquel Gobierno, q. no dará providencia ninguna acertada quando llegue el caso $=$ Que ademas tiene el poderoso partido de los Gobernadores Yndios, de los Teypanes de San Juan, y Santiago en Mexico y los de la(s) Provincias de Tlascala y Tépecaca, q. es el camino recto para Vera Cruz pr lo q. se conseguirán los efectos de cortar las remesas y toda correspondencia con Mexico = Que tiene tambien aviso de los Comisionados de California muy lisongeros, y q. no lo son menos los de Lima, q. cuenta el citado Desmolard segun los avisos q. ha recibido con los principales Xefes del Exercito particularmte con la guarnicion de Vera Cruz y con el destacamento del Castillo de 
Perote el qual tendrá luego pr suyo, siendo este un punto q. corta enteramte la correspondencia de todo el Reyno con Vera Cruz pr su situacion, y finalmte q. se lisongea de sus futuros proyectos = Es copia de la que existe en la Secretaria de la Suprema Junta Conservadora de los dros. del $\mathrm{Sr}$ dn Ferndo Septo en Venezuela; y po comunicar al Exmo. Sr Almte de Barbada en precaucion contra las asechanzas de Napoleon, firma la prete en Caracas á 31 de Moryo de 1810.

$$
\begin{aligned}
& \text { (fdo.) J. G. Roscio } \\
& \text { (una rúbrica) }
\end{aligned}
$$

\section{II}

Comisionados del Rey José Napoleon en las dos Americas

\section{(ff.188) Reyno de Mexico}

D. Antonio Renteria natural de San Sebastian en Vizcaya, Diputado en Xefe con destino en Mexico. Puebla, quatro Villas, Vera Cruz y costa hasta-Tampico,

D. Antonio Serrano, de Madrid en Acapulco, y costa del Sur de Nueva Espána, Eolima hasta San Blas.

D. Manuel Agudo de los Rios, Cordoves, en Valladolid, Selaya, Guanajato, y demas Ciudades inmediatas hasta Zacatecas.

D. Torquato Medina, Madrileño en Durango y todo el Reyno de la Nueva Vizcaya, incluso la costa desde la Vahia (sic) de San Bernardo el nuevo Santander hasta Tampico.

D. Anselmo Rodriguez natural de Cuenca en S. Luis Potosi.

D. Hipolito Mendieta, castellano viejo. Provincias internas, Sinaloa, y Chihuaque.

D. Sebastian Solorzano, Madrileño. Guadalajara, toda la nueva Galicia, y costa de San Blas, y Tepique.

D. Santiago Parreño, natural de la Coruña. 'Nuevo Mexico, y California alta y baxa.

D. Ygnacio Saldivar, Madrileño. Campeche, Tabasco y Soconuzco.

D. Esteban Romero. Granadino. Oaxaca Tehuantepeque. 


\section{Reyno de Guatemala.}

D. Estanislao Oropeza. Estremeño de Badajoz, Guatemala, Ornoa (?), y Provo de San Salvador. Xefe en este Reyno.

D. Ciriaco Betolaza de San Sebastian, Chiapa y Costa del Sur de Tonola hasta el Realejo.

D. Fermin Esparragosa en el Señorio de Truxillo, y toda la Prova de Comayahua.

D. Juan Chagaray. Vizcayno, en Leon de Nicaragua, Granada, Cartago, y Costa Rica hasta el Realejo, Sousanete, y Panamá.

\section{(ff.188v.) Reyno de Lima.}

D. Luis Azcarraja, Vizcaino. Lima y toda la costa hasta Guaryaquil: en Xefe en dho. Reyno.

Di: Cristoval Espinosa de Cordova en Quito.

D. Juan Viscarolaza. Vizcayno. Panamá hasta Portovelo, y la Costa hasta Guayaquil.

D. Remigio Aparicio de Victoria. Santiago de Chile.

D. Roque Frias. Madrileño. Provo de la Plata.

D. Benigno Alfaro, de Pamplona. Buenos Aires y Montevi. deo.

\section{Biblioteca de Letras}

\section{Reyno de Santap Fecinelli Converso»}

D. Cipriano Esparza. Estremeño. Santa Fé, Cartagena y costa hasta Portovelo, en Xefe.

D. Ermenegildo Estacheta. Vizcayno. Guayra, Caracas y costa de Cumaná.

D. Antonio Sanchez. Andalúz. Rio Lache y vecindario de su costa.

Han pasado ultimamente pa unirse con estos Comisionados otros venidos nuevamte de Europa. Trés pasaron pr la vía de Nueva Orleans á saber.

D. Juan Arevalo. Castellano viejo po juntarse con Parreño en nuevo Mexico, y California.

D. Mateo Cervantes. Madrileño, pa unirse, y estar á la disposicion de Mendieta en Provincias internas.

D. Raymundo Camarena. Madrileño po pasar a Mexico á la disposicion de Renteria. 


\section{Dos han ido á la Harvana que son} peche.

D. Bernardino Cisnéros del Reyno de Jaen pa pasar á Cam-

D. Lazaro Ybarzola vizcayno pa la Guayra.

Se embarcó otro po Cuba llamado D. Gregorio Anduaga, de Pamplona para pasar á Puertovelo en compañia de un mozo nombrado D. Pedro Banegas de la Alcarria.

En la Havana es el pral. un Español nativo de Bil (ff.189) bao qe pasa pr Americano. Su nombre legitimo es D. Ygnacio Berrechea. Este tiene mucho partido, y viaja la Ysla visitando sus Comisionados los qe se hallan esparcidos en varios puntos. Uno en Trinidad, dos en el Principe, uno en Santispiritus, uno en Villaclara, uno en la Villa de San Juan de los Remedios, otro en Holguin, otro en Baracoa, dos en Cuba, y otro en Matanzas.

En San Juan de Puerto Rico hay uno, y otro en la Aguada de San Franco y dos que viajan á Santa Cruz y Santo Tomas como contravandistas pa adquirir noticias pr esta via desde Curazao. Tienen de Caracas, y de toda la Costa Firme, Cartas de aquellos Comisionados.

En Jamayca hay tres pr cuya via tienen noticias repetidas, tanto del Reyno de Santa Fé, quanto del Perú por Puertovelo, como del Reyno de Bualêndia ya Nueva España go cuyos puntos navegan seis como contrabandistas Sotavento.

Hay dos Comisionados en Curazao, y uno en Trinidad de

En Charleston el correspondiente y comisionado es D. Juan Tineo natural de Valladolid.

D. Estanislao Morales, Castellano nuevo está en Nueva Orllego.

D. Santiago Antonino, y Mr. Lacroisee, ó St Croix han marchado en la Goleta Americana Fly po reunirse á Frias, y Alfaro en la Provincia de la Plata, Buenos Ayres, y Montevideo. Se cree tambien que ha ido á reunirse á los mismos Mr Duclos francés de edad 45 á 50 años qe ha sido Oficial de Marina al servicio de Carlos $4^{\circ}$ y actualmente ha entrado en clase de Capitan de $\mathrm{Na}$ vio al servicio de José.

Mr Leger Francés de las fronteras de Alemania, qe habla perfectamte los dos idiomas, y el Español, ha salido de Baitimore hace algunos dias po establecerse en las fronteras de la Luisiana, 
y hacerse partido entre los Alemánes establecidos en aquella Provincia con el obgeto de formár pr su medio una comunicacon con (ff.189v.) Mexico. Fue seguido de tres carruages cargados de mercancias y su proyecto es de establecerse como comerciante en Ovachita ó sus inmediaciones. Al despedirse del principal Agente Desmolard, le dixo este que siguiese las instrucciones que le habia dado.

Tres franceses han salido ultimamte po Augusta en la Georgia sin duda con animo de pasár desde alli á las Floridas. Sus nombres son Mrs Desmoyes, Corrolle, y Dinglin.

Tambien han marchado de Baltimore tres Españoles llarnados Manuel, Diego Sayo, y Domingo André, pero se ignona el camino que han tomado.

Un criollo de Lastibouitte en la Ysla de Santo Domingo ha pasado tambien á la Jamayca.

Es copia de la q. existe en la Secretaria de la Suprema Junta conservadora de los Dros. del Sr dn Fernando $7^{\circ}$ en Venezuela, y po comunicarla al Excmo Sr Almirte de la Barbada en precaucion de las acechanzas de Napoleon contra estos dominios, firmo la presente en Caracas á 1 de junio de 1810.

\section{(fdo.) J.G: Roscio
(una fúbrica) eca de Letras "Jorgé Puccinelli Converso"}

